\title{
ON THE QUANTIZATION OF CHARGED BLACK HOLES WITH ALLOWANCE FOR THE COSMOLOGICAL CONSTANT
}

\author{
M.G. Holovko*, V.D. Gladush \\ Oles Honchar Dnipro National University, Dnipro, Ukraine \\ *e-mail: dilmendar@gmail.com
}

\begin{abstract}
The paper considers a spherically symmetric configuration of the gravitational and electromagnetic fields with allowance to the cosmological constant, and its quantization. After dimensional reduction, the original action is transformed to new variables in the $R$ - and $T$-regions. The exclusion of the non-dynamic degree of freedom from the obtained action leads to an action for the geodesic in the configuration space, which proves to be conformally flat. We use the Gitman-Tyutin formalism for the obtained dynamical system, which Lagrange function is degenerate. After performing a suitable canonical transformation, the constraints found from the Lagrange function are reduced to the canonical form. Herewith the physical part of the Hamilton function vanishes. To construct quantum theory, we introduce additional physical quantities - charge and mass functions. Since Hamilton operator equals zero, it leads to the fact that the desired wave function of the system obeys only the eigenvalue equations for the mass and charge operators. The solution of these equations leads to continuous charge and mass spectra.

Keywords: charged black holes, cosmological constant, mass function, charge function, Hamiltonian constraint, quantization, mass and charge operators.
\end{abstract}

Received 03.11.2018; Received in revised form 10.12.2018; Accepted 21.12.2018

\section{Introduction}

Earlier, using Gitman-Tyutin method [1], in paper [2] the quantum model of a charged black hole $(\mathrm{BH})$ based on a spherically symmetric configuration of the electromagnetic and gravitational fields was considered. It was shown that in this model the mass and charge spectra were continuous. From the latest data from astrophysical observations of the global influence of dark energy on astrophysical structures, it would be interesting to consider this model taking into account the cosmological constant. Although the models under consideration belong to very small scales, the role of the cosmological constant for them is still unclear, especially in the early stages of the Universe. Basing on this, we consider the model of charged BH taking into account the cosmological constant, with using the GitmanTyutin method. Allowance for dark energy cardinally changes the global space-time and superspace structure of the considered configuration, the study of which turns out to be a difficult task. In this paper we offer the first results of the study. Subsequent results will be reviewed in further publications.

\section{Classic description of charged $B H$ with cosmological constant}

The total action for the gravitational, electromagnetic fields in space-time $V^{4}$ with cosmological constant $\Lambda$ has the form:

$$
S_{t o t}=-\frac{1}{16 \pi} \int_{V^{4}}\left\{\frac{c^{3}}{\kappa} R^{(4)}+\frac{1}{c} F_{\mu \nu} F^{\mu \nu}-2 \frac{c^{3}}{\kappa} \Lambda\right\} \sqrt{-g} d^{4} x
$$

Here $c$-velocity of light, $\kappa$ - gravitational constant, $R^{(4)}$ - scalar curvature of space-time, $F_{\mu \nu}$ - electromagnetic field tensor, $\Lambda$-cosmological constant, $g$-determinant of the metric tensor.

For a spherically symmetric configuration, the interval can be reduced to the form:

$$
d s^{2}=h\left(x^{0}, r\right)\left(d x^{0}\right)^{2}-g\left(x^{0}, r\right) d r^{2}-R^{2}\left(x^{0}, r\right) d \sigma^{2},
$$

where $d \sigma^{2}=d \theta^{2}+\sin ^{2} \theta d \varphi^{2}$. After integrating over the angles and discarding the surface term, the action (2.1) takes the form 


$$
S=\int_{V^{2}}\left(\frac{\left(A_{1,0}-A_{0,1}\right)^{2} R^{2}}{2 c \sqrt{g h}}+\frac{c^{3} \sqrt{g h}}{2 \kappa}\left[1+\frac{R R_{, 1}}{g}(\ln R h)_{, 1}-\frac{R R_{, 0}}{h}(\ln R g)_{, 0}-\Lambda R^{2}\right]\right) d^{2} x .
$$

Here $X_{, 0} \equiv \partial X / \partial x^{0}, X_{, 1} \equiv \partial X / \partial x^{1}$. Space-time of considered fields configuration consist of R- and T-regions. According to the Birkhoff theorem we can choose the coordinates in which the metric depends on the spatial coordinate $r$ in the R-regions and on the time coordinate $x^{0}$ in the T-regions:

$$
\begin{gathered}
d{s_{R}}^{2}=h(r)\left(d x^{0}\right)^{2}-g(r) d r^{2}-R(r) d \sigma^{2}, \\
d{s_{T}}^{2}=h\left(x^{0}\right)\left(d x^{0}\right)^{2}-g\left(x^{0}\right) d r^{2}-R\left(x^{0}\right) d \sigma^{2}
\end{gathered}
$$

Lagrange functions in this regions takes simple form:

$$
\begin{aligned}
& L_{R}=\frac{1}{2 c} \frac{A_{0,1}{ }^{2} R^{2}}{\sqrt{g h}}+\frac{c^{3}}{2 \kappa} \sqrt{g h}\left[1+\frac{R R_{, 1}}{g}(\ln R h)_{, 1}-\Lambda R^{2}\right], \\
& L_{T}=\frac{1}{2 c} \frac{A_{1,0}{ }^{2} R^{2}}{\sqrt{g h}}+\frac{c^{3}}{2 \kappa} \sqrt{g h}\left[1-\frac{R R_{, 0}}{h}(\ln R g)_{, 0}-\Lambda R^{2}\right] .
\end{aligned}
$$

In the R- and T- regions, it is convenient to go over to the new variables, respectively:

$$
\begin{array}{llll}
f_{R}=A_{0}, & u_{R}=\frac{1}{2} R(1+h), & n_{R}=\frac{1}{2} R(1-h), & N_{R}=\sqrt{g h}, \\
f_{T}=-A_{1}, & u_{T}=\frac{1}{2} R(1-g), & n_{T}=\frac{1}{2} R(1+g), & N_{T}=\sqrt{g h} .
\end{array}
$$

Then the Lagrange functions (2.6) and (2.7) take the uniform form:

$$
L_{\alpha}=\frac{1}{2 c} \frac{f_{, \alpha}{ }^{2}(u+n)^{2}}{N}+\frac{s}{2} N\left[1+\frac{u_{, \alpha}{ }^{2}-n_{, \alpha}{ }^{2}}{N^{2}}-\Lambda(u+n)^{2}\right] .
$$

Here $s=c^{3} / \kappa, \alpha$ - evolutionary parameter. Variable $\mathrm{N}$ in (2.9) is non-dynamical, and leads to the constraint $\partial L / \partial N=0$. Expressing $\mathrm{N}$ from Lagrange-Euler equations and substituting in (2.9), we get:

$$
S_{\text {sup }}=s \int \sqrt{\left(1-\Lambda(u+n)^{2}\right)\left(d u^{2}-d n^{2}+(u+n)^{2} d f^{2}\right)} .
$$

Thus, original action is reduced to the action for a geodesic in a three-dimensional pseudo-Riemannian space with the metric

$$
d \Omega^{2}=\left(1-\Lambda(u+n)^{2}\right)\left(-d n^{2}+d u^{2}+(u+n)^{2} d f^{2}\right) .
$$

Note that the resulting geodesic equations together with the constraint are equivalent to the Einstein equations for the metrics (2.4), (2.5). The metric can be reduced to conformally-flat type

$$
d \Omega^{2}=\left(1-\Lambda(a-b)^{2}\right)\left(-d a^{2}+d b^{2}+d w^{2}\right)
$$

by the following conversion of field variables:

4 


$$
u=\frac{w^{2}}{2(a-b)}-b, \quad n=a-\frac{w^{2}}{2(a-b)}, \quad f=\sqrt{s c} \frac{w}{a-b} .
$$

Lagrange function (2.9) describes degenerate system, so for further construction of canonic formalism we shall use Gitman-Tyutin method [1]. After substituting variables (2.12) in (2.9), we introduce Hamilton function of the system in extended phase space

$$
H=P_{i} V^{i}-L^{V} .
$$

Here $V^{i}=\dot{q}^{i}$ are generalized velocities corresponding to the generalized coordinates $q^{i}=\{a, b, w, N\}$. From the equations $P_{i}=\partial L / \partial V^{i}$ we obtain the velocities $V_{a}, V_{b}, V_{w}$, and primary constraint $P_{N}=0$. Substituting the velocities in (2.13) and calculating the Poisson bracket of the primary constraint with the Hamilton function, we find the secondary constraint

$$
\frac{1}{2 s}\left[-P_{a}^{2}+P_{b}^{2}+P_{w}^{2}-s^{2}\left(1-\Lambda(a-b)^{2}\right)\right]=0 .
$$

The Poisson brackets of the constraint (2.14) and the Hamilton function vanish, so there are no new constraints. The Poisson brackets of the found constraints also vanish, so they are second type constraints. It means that the system contains two non-physical degrees of freedom. For the further formalism construction it is necessary to explicitly separate physical and non-physical degrees of freedom. This is achieved by performing the canonical transformation to new variables $\left(q^{i}, p_{i}\right)$ with the following generating function of the second type [3]

$$
G_{2}\left(\alpha, Q^{i}, p_{i}\right)=N p_{4}+\frac{1}{2 p_{2}}\left[(a+b) p_{2}^{2}+(b-a)\left(s p_{3}+s^{2}\right)+\frac{\left(w p_{2}-p_{1}\right)^{2}}{b-a}+\frac{\Lambda s^{2}}{3}(a-b)^{3}\right] \text {. }
$$

In the new variables the constraints take the canonical form and the Hamiltonian function becomes equal to combination of constraints:

$$
H=p_{4} V_{N}+\frac{q^{4} p_{3}}{2} ; \quad p_{3}=p_{4}=0
$$

\section{Quantum description of a charged black hole}

By putting constraints in (2.16) equal to zero, we obtain that the physical part of the Hamilton function also equals to zero. Thus, in quantization, the wave function of the system is determined only by operators of physical quantities. In our case, these quantities are mass and charge of the $\mathrm{BH}$. In the classical case they are given by the relations

$$
Z=\frac{R^{2}}{\sqrt{g h}}\left(A_{0,1}-A_{1,0}\right) ; \quad M=\frac{s}{2 c} R\left(1+\frac{R_{, 0}{ }^{2}}{h}-\frac{R_{, 1}{ }^{2}}{g}-\frac{\Lambda}{3} R^{2}\right)+\frac{Z^{2}}{2 c^{2} R} .
$$

In variables $\left(q^{i}, p_{i}\right),(3.1)$ takes the form:

$$
Z_{p h}=\sqrt{\frac{c}{s}} p_{1} ; \quad M_{p h}=\frac{q^{2} p_{2}^{2}}{s c} .
$$


Solving the question of operator ordering for the mass operator from the requirement of operators to be Hermitian, we obtain the following operators of mass and charge

$$
\hat{Z}=-i \hbar \sqrt{\frac{c}{s}} \frac{\partial}{\partial q^{1}}, \quad \hat{M}=-\frac{\hbar^{2}}{s c} \frac{\partial}{\partial q^{2}} q^{2} \frac{\partial}{\partial q^{2}}
$$

By solving the system of eigenvalue equations for operators (3.3), we obtain the desired wavefunction for the considered field configuration

$$
\Psi\left(q^{1}, q^{2}\right)=C J_{0}\left(\frac{2}{\hbar} \sqrt{s c m q^{2}}\right) \exp \left(\frac{i}{\hbar} \sqrt{\frac{s}{c}} z q^{1}\right)
$$

Thus, in the considered model, the charge and mass spectra are continuous, which is consistent with the papers [3 - 7]. The corresponding expression completely coincides with the result of [3] for a neutral $\mathrm{BH}$, which indicates the correctness of the used method. Note also that the obtained result does not explicitly depend on $\Lambda$, which makes it similar to the result of [7] for an ordinary charged BH. However, these solutions are not identical, since in this paper the configuration space is conformally flat, but not flat as in [7].

\section{Conclusion}

Thus, the application of the method by D.M. Gitman and I.V. Tyutin to build a model of a charged $\mathrm{BH}$ leads to continuous mass and charge spectra. The presence of the cosmological constant in this approach does not have an obvious effect on the wave function of the system. However, it leads to the fact that the configuration space becomes conformally flat. The obtained result is consistent with previous papers [2 -7], and also corresponds to the results of papers $[4,8]$ for a $\mathrm{BH}$ without a cosmological constant.

\section{References}

1. Gitman, D.M. Kanonicheskoye kvantovanie system so svyazyamy / D.M. Gitman, I.V. Tutin // M.: Nauka, 1986. - 216 p.

2. Holovko, M.G. On the quantization of charged black holes / M.G. Holovko, V.D. Gladush // Vìsnik Dnìpropetrovs'kogo unìversitetu, Seria Fìzika, radioelectronika. 2016. - Vol. 25, Issue 24. - P. 3 - 6.

3. Gladush, V.D. On the quantization of black holes / V.D. Gladush // Vìsnik Dnìpropetrovs'kogo unìversitetu, Seria Fìzika, radioelectronika. - 2016. - Vol. 24, Issue 23(2). - P. $31-34$.

4. Kuchar, K. Geometrodynamics of Schwarzschild black holes / K. Kuchar. // Phys. Rev. D. - 1994. - Vol. 50. - P. 3961 - 3981.

5. Louko, J. Hamiltonian thermodynamics of the Reissner-Nordstrom anti-de Sitter black hole / J. Louko, S. Winters-Hilt // Phys. Rev. D. - 1996. - Vol. 54. - P. 2647 - 2663.

6. Nakamura, K. Quantum Fluctuations of Black Hole Geometry / K. Nakamura, S. Konno, Y. Oshiro, A. Tomimatsu // Prog. Theor. Phys. - 1993. - Vol. 90 (4). - P. 861 -870 .

7. Holovko, M.G. Quantization of spherically symmetric gravitational field / M.G. Holovko, V.D. Gladush // International Conference "Astronomy and Space Physics". Abstract book. - Kiev, Ukraine, 23 - 26 May 2017. - P. 31.

8. Holovko, M.G. Quantization of charged black holes as degenerate systems / M.G. Holovko, V.D. Gladush // International Conference "Astronomy and Space Physics". Abstract book. - Kiev, Ukraine, 29 May - 1 June 2018. - P. 31. 